\title{
Emicizumab, a humanized bispecific antibody to coagulation factors IXa and $X$ with a factor VIIla-cofactor activity
}

\author{
Takehisa Kitazawa ${ }^{1} \cdot$ Midori Shima $^{2}$
}

Received: 18 July 2018 / Revised: 7 September 2018 / Accepted: 10 September 2018 / Published online: 22 October 2018

(c) The Japanese Society of Hematology 2018

\begin{abstract}
Hemophilia A is a congenital disorder caused by deficiency or malfunction of coagulation factor (F) VIII. While exogenously provided FVIII effectively reduces bleeding complications in many hemophilia A patients, multiple efforts are underway to develop new drugs to meet the needs that conventional FVIII agents do not. We have been long engaged in creating and clinically developing a humanized anti-FIXa/FX asymmetric bispecific IgG antibody with a FVIIIa-cofactor activity. Since this project was born from a creative and unique idea, our group recognized from the first that it would face many difficulties in the course of research including establishment of industrial manufacturability of an asymmetric bispecific IgG antibody. The group actually faced various challenges, but addressed all of them during about 10 years of research, and successfully created the potent humanized bispecific antibody, emicizumab. Emicizumab has showed clinical benefits in the human trials among which the first one was started in 2012, and has been currently approved in US, EU, Japan, and some other countries. It is now expected to improve the quality of life of patients and their families. In this article, we review the course of the research and clinical development of emicizumab, and describe its molecular characteristics.
\end{abstract}

Keywords Bispecific antibody $\cdot$ Emicizumab $\cdot$ Factor VIII $\cdot$ Hemophilia A

\section{Introduction}

Hemophilia A is a congenital disorder caused by deficiency or malfunction of coagulation factor (F) VIII. In approximately half of hemophilia A patients, the disease is severe [1], which is defined as $<1 \%$ of normal FVIII activity [2]. Patients with severe hemophilia A typically experience frequent spontaneous bleedings into their joints and/or muscles, predominantly in the absence of identifiable hemostatic challenge. The gene encoding FVIII ( $F 8$ gene) is located on $\mathrm{X}$ chromosome, and consequently, most of the patients with severe hemophilia A are male.

The first line of treatment for severe hemophilia A is FVIII replacement therapy. This treatment against ongoing

Takehisa Kitazawa

kitazawatkh@chugai-pharm.co.jp

1 Research Division, Chugai Pharmaceutical Co., Ltd., 1-135 Komakado, Gotemba, Shizuoka 412-8513, Japan

2 Department of Pediatrics, Nara Medical University, Kashihara, Nara, Japan bleeding episodes has decreased the mortality caused by bleeding [3]. In addition, on the basis of the observation that moderate hemophilia A patients (1-5\% of normal FVIII activity) experience far fewer bleeding episodes, many patients with severe hemophilia A in developed countries are currently given regular prophylactic infusions of a FVIII agent to keep the factor's activity to $1 \%$ or above of normal activity, which effectively reduces joint bleeds, maintains better joint status, and leads to an improved quality of life $[2,4]$.

Despite these advantages of the FVIII replacement therapy, there remain some unmet issues [5]. Since the plasma half-life of a FVIII molecule is rather short (approximately 0.5 days), frequent infusions, typically three times a week, are required for bleeding prophylaxis [2, 4]. Currently, FVIII agents with an extended half-life have appeared on the market, but the plasma half-life is around 0.75 days, so once or twice weekly infusions are still required [6]. In addition, as exogenous FVIII is only available by intravenous infusions [7], the difficulty of venous access is another big hurdle for introducing injections in the home setting. Furthermore, as the FVIII molecule is immunologically a foreign protein 
in hemophilia A patients, $1 / 3$ of severely affected patients develop anti-FVIII neutralizing alloantibodies, termed FVIII inhibitors [8], which decrease or diminish the effect of a FVIII agent. If FVIII inhibitors develop, it becomes more difficult to control hemorrhaging, because alternative treatment agents, including recombinant activated FVII and activated prothrombin complex concentrates (aPCC), have a shorter half-life and are not always effective [2]. Eradication of FVIII inhibitors with high doses of a FVIII agent is also being attempted (termed immune tolerance induction or ITI), but the process is burdensome and does not always work [9]. From the economic point of view, the treatment cost for hemophilia is also a big issue especially in developing countries.

To overcome the remaining needs of patients with severe hemophilia A, a number of researchers in academia and industry are continuing the struggle. Besides our approach that will be described in detail later [10], multiple approaches are under clinical development [5, 6]. One example is a further engineered FVIII molecule, BIVV001, which is currently in phase $1 / 2$ of clinical development and is reported to have approximately 1.5 days of half-life instead of the 0.75 days achieved by a current FVIII agent with an extended half-life. The BIVV001 agent is aimed at reducing the frequency of infusions, but still needs venous access.

Another type of therapy that uses the approach of "rebalancing" the hemostatic mechanism is the use of an inhibitor against the innate anticoagulation pathway: for example, siRNA against antithrombin (fitusiran) under clinical development in phase 3 , and anti-tissue factor pathway inhibitor (TFPI) antibodies (concizumab etc.) under clinical development in phase 1 or 2 . These agents can be given in subcutaneous injections instead of intravenous infusions and thus have the advantage of dosing route, as well as possibly the advantage of reducing dosing frequency. In addition, they will be able to address the issue of FVIII inhibitors, since their antigenicity is totally different from that of a FVIII molecule.

Gene therapy has been long awaited as an ultimate therapy. Currently, multiple types of $F 8$ gene transfer mediated by the recombinant adeno-associated virus (AAV) are being clinically developed in phase $1 / 2$ or 3 . Clinically meaningful outcomes have been recently reported, and thus, this fantastical approach is gradually advanced, although several issues remain, such as anti-AAV neutralizing alloantibodies, therapeutic durability, and the use in patients with FVIII inhibitors [11].

Among these various approaches, a humanized antiFIXa/FX bispecific antibody with a FVIIIa-cofactor activity, emicizumab (Hemlibra ${ }^{\circledR}$ ) [12], was first approved and introduced to the market in US, EU, Japan, and some other countries. In this review article, we describe the course of the research and the clinical development of emicizumab, as well as its molecular characteristics.

\section{Inspiration for an anti-FIXa/FX bispecific antibody with a FVIIla-cofactor activity}

The concept of an anti-FIXa/FX bispecific antibody with a FVIIIa-cofactor activity (Fig. 1a) was inspired by Dr. Kunihiro Hattori, a researcher in Chugai Pharmaceutical Co., Ltd. (Chugai) around the year 2000. In those days, his group was engaged in several therapeutic antibody projects, one of which was a humanized anti-tissue factor antibody for treating thrombotic disorders, which meant that he had extensive knowledge of both antibodies and coagulation. He had noticed reports in the mid-late 1990s, indicating that
Fig. 1 Mechanisms of actions of FVIIIa-cofactors. a Schematic illustration for the idea of antiFIXa/FX bispecific antibody with a FVIIIa-cofactor activity. b Schematic illustration for FVIIIa binding to FIXa and FX on the basis of published reports [13-16, 18, 19]. PS phosphatidylserine, $P L$ phospholipid, $E G F 1,2$ epidermal growth factor-like domain 1,2
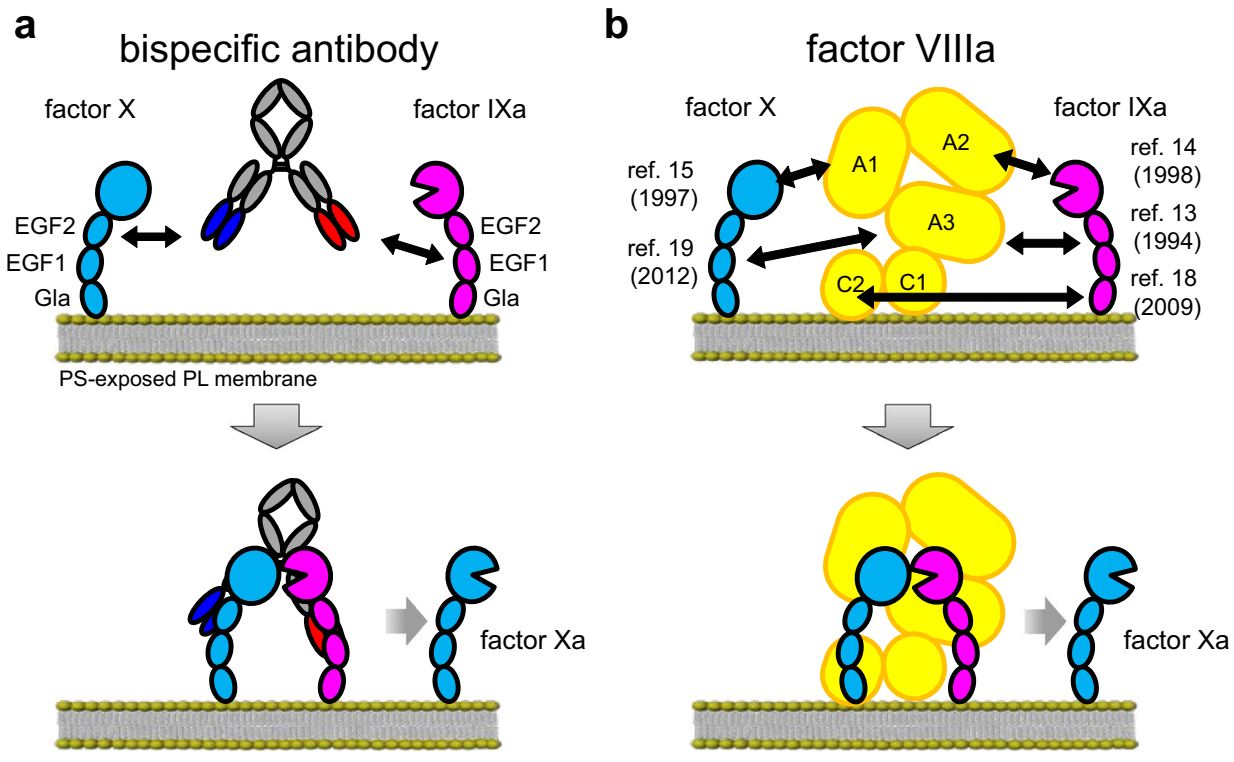
FVIIIa had binding abilities to both FIXa and FX (Fig. 1b) [13-16]. He was also aware of the report in the late 1990s that revealed the molecular mechanism of Protein S cofactor function to accelerate the activated Protein $\mathrm{C}$ (aPC)catalyzed "complete" inactivation of FVa [17]. The report demonstrated that protein $\mathrm{S}$ facilitated rotational and/or translational movement of the aPC catalytic center relative to the cell surface at the same level of the R306 position of FVa, and thus improved the accessibility of the center to the R306 position at which cleavage led to "complete" inactivation of FVa. Without Protein S, the position of the aPC catalytic center relative to the cell surface was at the same level of the R506 position of FVa at which cleavage led to "incomplete" inactivation of FVa. In other words, the function of Protein $\mathrm{S}$ cofactor is simply changing the spatial positioning of the aPC catalytic center above the cell surface to control the intensity of the aPC-dependent negative feedback of coagulation. Having these background, he inspired that there could be an anti-FIXa/FX bispecific IgG antibody to exert a FVIIIa-cofactor activity by placing the catalytic center of FIXa precisely at the cleavage site of FX, although such an antibody would be rare [10]. An IgG antibody generally has a high subcutaneous bioavailability and a long half-life. In addition, an IgG antibody would have a totally different structure or antigenicity from the FVIII molecule, so it should theoretically work even in the presence of FVIII inhibitors and also would not itself induce FVIII inhibitors. These were ideal drug profiles for hemophilia A.

FVIIIa is a cofactor to increase the catalytic rate constant $\left(k_{\text {cat }}\right)$ and decrease the Michaelis constant $\left(K_{\mathrm{m}}\right)$ of the reaction of FIXa-catalyzed FX activation [10]. Although pioneering researchers had revealed that multiple sites in FVIIIa interact with FIXa and FX already at that time (Fig. 1b) [13-16], the mechanisms by which FVIIIa accelerates the FIXa-catalyzed FX activation had yet to be defined even at the time of writing. FVIIIa may facilitate the precise placement of the catalytic center of FIXa at the cleavage site of FX by its ability to bind to both factors. In addition, the A2 subunit of FVIIIa may directly modulate the active catalytic site of FIXa to increase $k_{\text {cat }}$ of the FIXa-catalyzed FX activation [14]. The strategy of applying a bispecific antibody would realize only the binding to the respective antigens at single sites, and therefore, it was a great challenge to reproduce such a complicated original FVIIIa-cofactor function facilitated by multiple interactions. Nevertheless, our attempts were encouraged by the epidemiologic observation that just keeping only a few $\%$ of normal FVIII activity would be enough to prophylactically reduce the bleeding episodes of patients with severe hemophilia A [2]. In terms of molecular biology, the group also perceived that the distance between the FIXa- and FX-binding sites of FVIIIa would be similar to that between the two antigen-binding sites of human IgG [10, 20, 21].
In terms of developing a bio-pharmaceutical, another critical issue at that time was how to manufacture an asymmetric bispecific IgG antibody. Since an IgG antibody has two antigen-binding fragments (Fab) both of which link to an $\mathrm{Fc}$ region, there had long been a concept of an asymmetric bispecific IgG antibody capable of binding to two different antigens or epitopes (Fig. 2a). Even now, however, only two asymmetric bispecific IgG antibody drugs have been approved in US, EU, or Japan so far. One is catumaxomab $\left(\right.$ Removab $\left.^{\circledR}\right)$, a rat-mouse hybrid asymmetric bispecific IgG antibody to CD3 and epithelial cell adhesion molecule (EpCAM), which was approved in EU in 2009 but withdrawn in 2017. The other is emicizumab (Hemlibra ${ }^{\circledR}$ ), our humanized asymmetric bispecific IgG antibody to FIXa and FX. An asymmetric bispecific IgG antibody typically has 2 kinds of heavy chain and 2 kinds of light chain. If the 4 chains were simultaneously expressed in a recombinant cell to produce the desired asymmetric bispecific IgG antibody, as many as 9 kinds of unwanted byproduct IgG molecules would also be assembled and secreted (Fig. 2b). This would lead to difficulties in the purification process in industrial manufacture, as well as decreasing the production efficiency. In 1998, researchers at Genentech Inc. threw some light on this issue [22]. They advocated a method of reducing the byproduct IgG molecules using a common light chain in the asymmetric bispecific IgG antibody and also by introducing "knobs-into-holes" mutations in its Fc region to prioritize a specific hetero-dimerization of the heavy chains. This report encouraged us in terms of pharmaceutical technology, although difficulties in the industrial manufacture still laid ahead.

\section{Generation and molecular engineering of the bispecific antibody}

The bispecific antibody we were trying to create had to place the catalytic center of FIXa precisely at the cleavage site of FX. As we mentioned above, we considered that such a bispecific antibody should be rare, if any. Therefore, our strategy was to create a large number of anti-FIXa/FX bispecific antibodies to discover just the "rare" one we needed $[10,12]$. In the early era of this program, we identified, from a screening of 460 generated anti-FIXa/FX bispecific antibodies, an anti-FIXa/FX bispecific antibody that shortened the activated partial thromboplastin time (APTT) of FVIIIdeficient plasma to a certain extent. Soon after that, the Chugai members visited Nara Medical University, a prominent hemophilia research group in Japan, to discuss the potency and prospects of the anti-FIXa/FX bispecific antibody, and then the collaboration between the two institutions started. The collaboration is now turned out to be continued beyond a decade. 
a

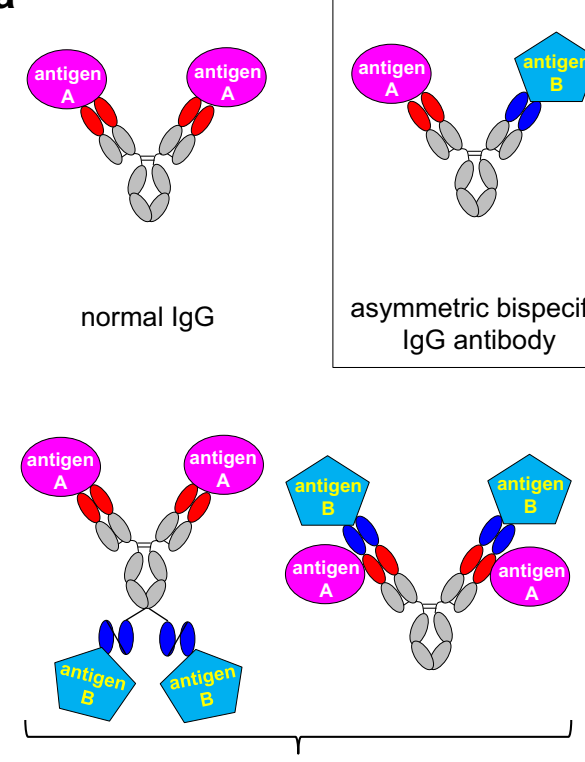

symmetric bispecific antibodies (examples) b

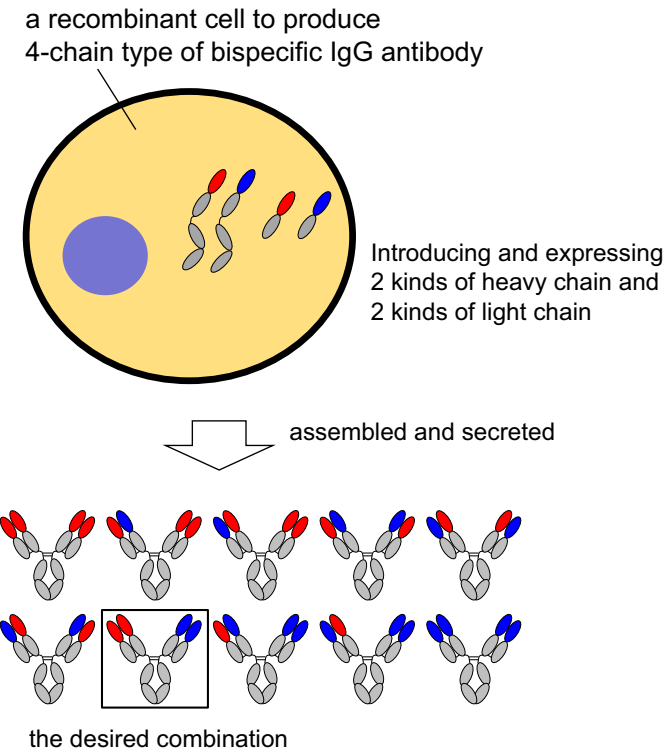

c emicizumab molecule

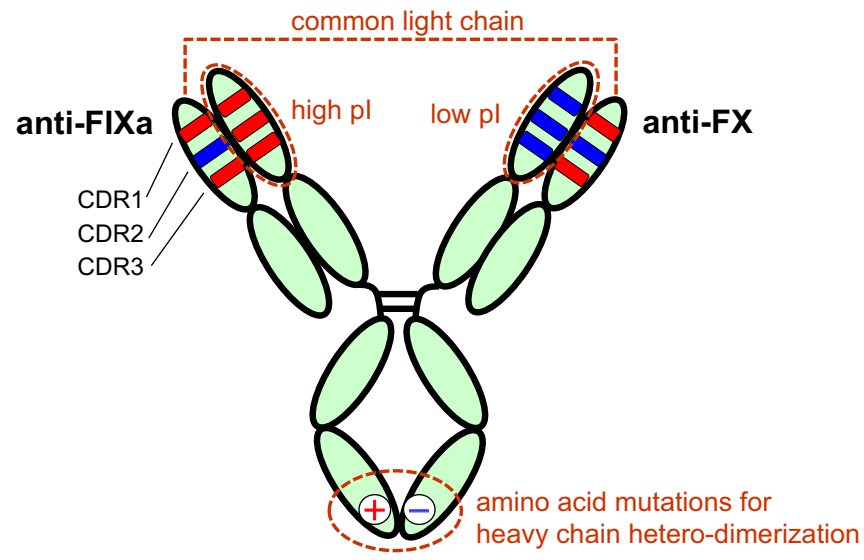

derived from a rat anti-human FIXa antibody

derived from a mouse anti-human FX antibody

derived from a human $\lg \mathrm{G}_{4} / \kappa$

Fig. 2 Information on bispecific antibodies and molecular structure of emicizumab. a Schematic illustrations of an asymmetric bispecific IgG antibody and some examples of symmetric bispecific antibodies. b Illustration of a major problem for industrially manufacturing an asymmetric bispecific IgG antibody. When 2 kinds of heavy chain and 2 kinds of light chain were expressed in a recombinant cell to

For some time, our research activities were just like trial and error. For example, we first considered that the APTTshortening effect would reflect the in vivo hemostatic activity of the bispecific antibodies in the hemophilia A state, as is the case with FVIII. During the course of our early research activities, we steadily accumulated knowledge on produce the desired asymmetric bispecific IgG antibody, as many as 9 kinds of unwanted byproduct IgG molecules would be simultaneously assembled and secreted. c Schematic illustration of the molecular structure of emicizumab. $C D R$ complementarity determining region, $p I$ isometric point

the true nature of FVIIIa-cofactor activity of the bispecific antibodies, and became aware that an APTT-shortening activity would not necessarily predict the in vivo hemostatic activity.

We repeated the screening campaigns, and eventually, the screening scale was expanded to tens of thousands of 
bispecific antibodies to identify a lead bispecific antibody with "good natures". Briefly, we prepared the genes of the variable regions of anti-FIXa or anti-FX antibodies (approximately 200 for each antigen) from mice, rats, and rabbits that had been immunized with human FIXa or FX. We inserted the genes into an expression vector containing the constant region of human $\mathrm{IgG}$, and expressed a total of approximately 40,000 bispecific IgG combinations in Human Embryonic Kidney (HEK) cells. The FVIIIa-cofactor activity of each bispecific antibody was tested one by one in an enzymatic assay consisting of purified FIXa and FX, synthetic phospholipid (PL), and a chromogenic substrate for FXa. The bispecific antibodies selected from the mass screening were further narrowed down by a series of assays, including plasma assays. Then, we identified a lead anti-FIXa/FX bispecific antibody, BS15, with 2 kinds of heavy chain and a common light chain, and its humanized version, hBS1 [12]. The FVIIIa-cofactor activity of these antibodies, however, seemed to be insufficient for clinical use. In addition to the need to increase the FVIIIa-cofactor activity, we also had to multi-dimensionally improve other aspects: for example, to increase the physicochemical stability (necessary for storage stability), to increase the solubility (necessary for preparing a subcutaneously injectable formulation of high concentration), to decrease the non-specific binding (necessary for ensuring a subcutaneous bioavailability), and to improve the in silico prediction score on immunogenicity. For achieving all of these, we had to engineer the bispecific antibody molecule by replacing amino acids in the Fab regions. In the actual engineering process, we repeated an "engineering cycle" to gradually bring the designed antibody sequence closer to the goal. Briefly, in each "engineering cycle", we first designed several tens of bispecific antibody sequences by introducing amino acid mutations. Then, we expressed and purified the antibodies, and tested them multi-dimensionally to find promising amino acid mutations. However, an amino acid mutation that was promising for one or more aspects did not necessarily improve all of them. In such a case, we tried to design antibodies that would retrieve the aspects made worse by the previous mutations in the later cycles.

In addition, we also had to ensure the manufacturability of a recombinant asymmetric bispecific IgG antibody. As described above, if the 2 kinds of heavy chain and 2 kinds of light chain were expressed in a recombinant cell to produce the desired asymmetric bispecific IgG antibody, 9 kinds of unwanted byproduct IgG molecules would also be assembled (Fig. 2b). To resolve this issue completely and to manufacture at a high quality, we developed three proprietary technologies: a methodology to identify a common light chain effective for both the heavy chains of an asymmetric bispecific IgG antibody; isometric point (pI) engineering of the heavy chains to enable the desired bispecific IgG antibody to be isolated from the unwanted byproduct IgG molecules; and mutations to facilitate heavy chain hetero-dimerization by electric repulsion and attraction [12].

After testing as many as approximately 2,400 bispecific antibodies that we designed, we finally identified a promising humanized anti-FIXa/FX asymmetric bispecific IgG antibody, ACE910, which incorporated the proprietary technologies for industrial manufacturing and met all of the required aspects [12]. The international nonproprietary name of ACE910 was determined later as emicizumab.

\section{Non-clinical characteristics of emicizumab}

The origins of the complementarity determining regions (CDRs) in the anti-human FIXa heavy chain, the anti-human FX heavy chain, and the common light chain of emicizumab are rat, mouse, and both rat and mouse, respectively [12] (Fig. 2c).

Emicizumab surely functions as a cofactor to promote the FIXa-catalyzed FX activation, since it did not promote FX activation without FIXa in an enzymatic assay. For emicizumab to exert a FVIIIa-cofactor activity, it must simultaneously bind FIXa and FX; in other words, it must bridge FIXa and FX. This feature was demonstrated by the experimental result that neither the monospecific antibodies to FIXa or FX derived from emicizumab nor a mixture of those derived antibodies promoted FIXa-catalyzed FX activation [12]. Dependency of emicizumab's action on phosphatidylserine (PS)-exposed PL membrane is also important for addressing the site specificity of its action. The coagulation reaction is known to be dependent on PS-exposed PL membrane. This dependency contributes to limiting the fibrin formation to the hemostatic site, where platelets are activated to expose PS on their PL membrane surface. Whereas FVIIIa is known to have domains that bind to PS, emicizumab does not (Fig. 1), but nevertheless, the FVIIIa-cofactor activity of emicizumab was dependent on PS-exposed PL membrane [23]. This is because the FVIIIa-cofactor action in nature takes over the PS-exposed PL membrane dependency that the reaction of FIXa-catalyzed FX activation itself has.

The in vitro FVIIIa-cofactor activity of emicizumab was also demonstrated in a series of assays using FVIII-deficient or depleted plasma or blood to which emicizumab was added $[12,24,25]$. In addition, an in vivo hemostatic activity of emicizumab was demonstrated in an acquired hemophilia A animals. Since emicizumab is highly species specific, we established an acquired hemophilia A state in cynomolgus monkeys by injecting an anti-primate FVIII neutralizing antibody that did not cross react to porcine FVIII [24, 26]. Against the ongoing bleeding induced artificially in the animals, intravenous single administration of $3 \mathrm{mg} / \mathrm{kg}$ of emicizumab demonstrated a hemostatic activity similar to 
twice daily intravenous injections of $10 \mathrm{U} / \mathrm{kg}$ of recombinant porcine FVIII [24]. Based on this result, we roughly predicted that the factor to convert $\mu \mathrm{g} / \mathrm{mL}$ of emicizumab to the equivalent IU/dL of FVIII hemostatic activity would be 0.3 when the concentration of emicizumab in plasma was around $50 \mu \mathrm{g} / \mathrm{mL}[24,27,28]$. Later on, the prediction from non-clinical in vivo study turned out to be roughly concordant with the outcomes of cohort 2 in the phase 1 study which suggested that the factor would be 0.3 or higher for the conversion of $\mu \mathrm{g} / \mathrm{mL}$ of emicizumab to IU/dL of equivalent FVIII hemostatic activity [27].

The experimental results on binding properties of emicizumab are very useful for considering its mechanism of action. Emicizumab recognizes the epidermal growth factor (EGF)-like domain 1 of FIX/FIXa with one arm and the EGF-like domain 2 of FX/FXa with the other arm [23]. Thus, emicizumab does not bind directly to the pro-catalytic or catalytic domains of FIX/FIXa or FX/FXa. Its levels of binding affinity to the antigens are moderate (the $K_{\mathrm{D}}$ values are: $1.58,1.52,1.85$, and $0.978 \mu \mathrm{M}$ to FIX, FIXa, FX, and FXa, respectively) compared to typical therapeutic antibodies that have been marketed so far; for example, $0.0273 \mathrm{nM}$ (infliximab), $0.120 \mathrm{nM}$ (eculizumab), and $3.06 \mathrm{nM}$ (nivolumab). Emicizumab's moderate binding affinity, more specifically fast $k_{\text {off }}$, allows the resultant FXa to be released from emicizumab and move to the next downstream reaction in the coagulation cascade [23].

The $K_{\mathrm{D}}$-based simulation predicted that, at the plasma concentrations of emicizumab in the clinical setting, the majority of plasma FIX and FX would exist as monomers unbound to emicizumab in circulation [23]. This prediction suggests that any influence of emicizumab in the clinical setting on the other coagulation reactions would be minimal.
The $K_{\mathrm{D}}$-based simulation also predicted that only a very small part of plasma FIX, FX, and emicizumab would form the antigen-bridging FIX-emicizumab-FX ternary complex in circulation, and the concentration of the ternary complex would form a bell-shaped relationship with emicizumab concentration (Fig. 3a). The plasma emicizumab concentration at the peak of the bell shape was calculated as $265 \mu \mathrm{g} / \mathrm{mL}$ or $1820 \mathrm{nM}$ [23], whereas the steady-state emicizumab concentration in plasma with weekly $1.5 \mathrm{mg} / \mathrm{kg}$ subcutaneous injections in clinical was around $50 \mu \mathrm{g} / \mathrm{mL}[28,29]$. In the plasma thrombin generation assay using FVIII-deficient plasma, we found a similar bell-shaped relationship with emicizumab concentration (Fig. 3b), suggesting that emicizumab's cofactor activity depends on its ability to bridge the antigens [23]. Therefore, on the basis of the above assumption, we consider that too much emicizumab would not necessarily lead to too much cofactor activity. It may be preferable, because an unexpectedly high FVIIIa-cofactor activity would not be induced by emicizumab injections. Nevertheless, a higher emicizumab concentration in plasma (e.g., $200 \mu \mathrm{g} / \mathrm{mL}$ or more) should be considered with caution, since it would decrease emicizumab-unbound FIX and FX monomers in circulation.

We also performed a kinetic analysis to quantitatively elucidate the nature of emicizumab's cofactor activity. In the reaction of FIXa-catalyzed FX activation in the presence of synthetic PL, emicizumab increased $k_{\text {cat }}$ by 4,480 -fold and decreased $K_{\mathrm{m}}$ by 19.5 -fold, compared with the condition in the absence of cofactor [23]. The $k_{\text {cat }}$ value for the FIXacatalyzed FX activation in the FIXa-FVIIIa-FX ternary complex was 126 min and that in the FIXa-emicizumab-FX ternary complex was $2.88 \mathrm{~min}$. Thus, there was theoretically as much as 43.8 -fold difference in the rate (speed) of
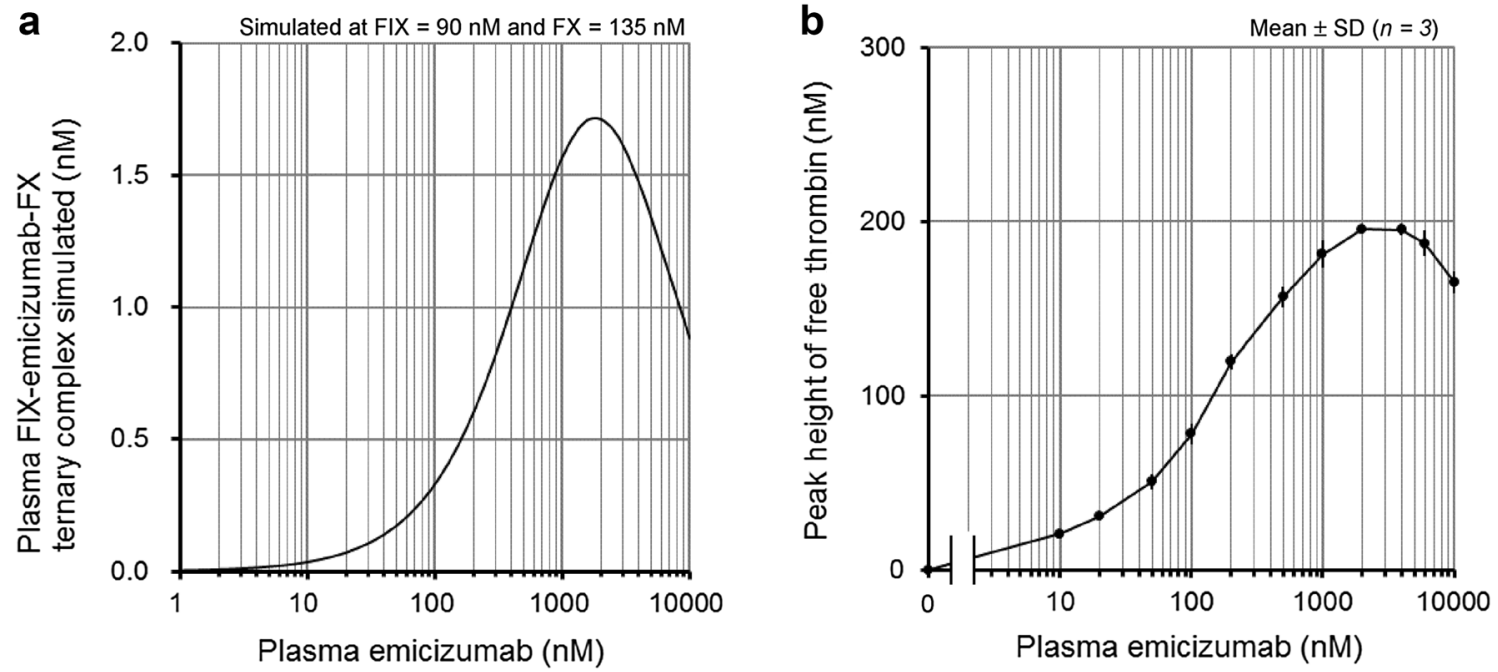

Fig. 3 Bell-shaped relationships with plasma emicizumab concentration of a simulated FIX-emicizumab-FX ternary complex formation in circulation and $\mathbf{b}$ thrombin generation potential of FVIII-deficient plasma 
FX activation between them, while plasma emicizumab at around $50 \mu \mathrm{g} / \mathrm{mL}$ is expected to provide an equivalent FVIII activity of at least $15 \mathrm{IU} / \mathrm{dL}$ or one 6.67 th of normal activity in vivo, as mentioned above [28]. These independent analyses may seem discrepant, but are assumedly logical when it is remembered that as the $K_{\mathrm{D}}$-based simulation showed, approximately $1 \mathrm{nM}$ of ternary complexes would be formed at around $50 \mu \mathrm{g} / \mathrm{mL}(343 \mathrm{nM})$ of plasma emicizumab, whereas the molar concentration of $15 \mathrm{IU} / \mathrm{dL}$ of FVIII is only $0.045 \mathrm{nM}$. Thus, we consider that the larger amount of enzyme-cofactor-substrate complexes in case of emicizumab would compensate for the lower rate (speed) of FX activation when comparing with the FVIII case [23].

Possibility of influence of emicizumab on the innate negative feedback systems of coagulation also needs to be clarified. Emicizumab interfered with the action of neither antithrombin nor TFPI [30], which means that the coagulation negative feedback by antithrombin and TFPI would fully work in the emicizumab-mediated coagulable reactions. Regarding the Protein C system, although emicizumab was not a substrate of aPC, as FVIIIa was, the Protein C system worked well via FVa inactivation to stop the emicizumab-mediated coagulable reaction in the plasma thrombin generation assay [31].

\section{Outcomes of the clinical studies of emicizumab}

The phase 1 study of emicizumab was initiated in 2012. It started with a double-blind, randomized, placebo-controlled, inter-individual dose-escalation part in healthy male adults for assessing the safety, tolerability, pharmacokinetics, and pharmacodynamics [32]. A total of 40 Japanese (Part A) and 24 Caucasian (Part B) subjects received a single subcutaneous injection of emicizumab (Part A: 0.001, 0.01, $0.1,0.3$, and $1 \mathrm{mg} / \mathrm{kg}$; Part B: $0.1,0.3$, and $1 \mathrm{mg} / \mathrm{kg}$; and 6 subjects per dose group) or placebo (2 subjects per dose group). In both parts of the study, emicizumab exhibited a linear pharmacokinetic profile and had a half-life of approximately 4-5 weeks (28.3-34.4 days). There was no serious adverse event, and no clinical or laboratory evidence of hypercoagulability-related abnormality. Anti-emicizumab alloantibodies, which are anti-drug antibodies (ADAs), were detected before and after emicizumab administration in one individual, and an ADA-positive test was reported only after administration in another subject.

Subsequently, an open-label, non-randomized, inter-individual dose-escalation part of emicizumab started in 2013 in 18 Japanese patients with severe hemophilia A ( $\geq 12$ years) with and without FVIII inhibitors (Part C) [27], and seamlessly, its extension study (the phase $1 / 2$ study) started in 16 of the 18 patients [33]. The purpose of the part and study was to assess the safety, pharmacokinetics, pharmacodynamics, and prophylactic effects on bleeding compared with the 6 months before enrollment in an exploratory manner. The dosing regimens-subcutaneous administrations at an initial dose of $1(\mathrm{C}-1)$ or $3(\mathrm{C}-2$ and $\mathrm{C}-3) \mathrm{mg} / \mathrm{kg}$ followed by a once-weekly dose of 0.3 (C-1), 1 (C-2), or 3 (C-3) mg/ $\mathrm{kg}$ - were determined on the basis of the non-clinically predicted concentrations of emicizumab in plasma at 1 week after each administration (trough levels) of 11.2 (C-1), 37.3 (C-2), and 112 (C-3) $\mu \mathrm{g} / \mathrm{mL}$ at steady state [27]. Since the non-clinically estimated factor for the conversion of $\mu \mathrm{g} / \mathrm{mL}$ of emicizumab to IU/dL of equivalent FVIII hemostatic activity was 0.3 as explained above, we expected that the outcomes may roughly correspond to a moderate $(\mathrm{C}-1)$ or mild (C-2 and 3) phenotype of hemophilia A [27].

Once-weekly subcutaneous emicizumab administration for a maximum of 33.3 months was well-tolerated with no thromboembolic event reported, even at the steady state for the highest dose ( $3 \mathrm{mg} / \mathrm{kg}$ per week), and no neutralizing ADA developed during the course of the part and study. Four patients tested positive for non-neutralizing ADAs: 1 tested positive before and after emicizumab initiation, and 3 tested positive only after emicizumab initiation. Because the presence of ADAs had no effect on plasma concentrations of emicizumab, FIX or FX, pharmacodynamic markers, or reduction in efficacy, the ADAs were considered non-neutralizing ones. In two patients, the treatment was discontinued. The reason in one case was repeated mild injection-site erythema. The other case did not meet one of the pre-determined criteria for participating in the extension phase 1/2 study: 3 or more bleeding episodes during the 6 months before enrollment in the case of a patient without FVIII inhibitors.

The emicizumab treatment in these studies showed encouraging efficacy in patients with severe hemophilia A, irrespective of the presence of FVIII inhibitors, as demonstrated by the substantial decrease in annualized bleeding rate (ABR) across dose cohorts (Fig. 4a). In a small subset of 4 patients, the dose was increased because of suboptimal bleeding control. In each patient who received higher doses, a further reduction in ABR was observed (Fig. 4b). These clinical outcomes seemed to be roughly concordant with our non-clinical estimate of the equivalent FVIII hemostatic activity of emicizumab described above. In other words, in this case, a non-clinical estimate was effectively utilized in the clinical study design (the dosage setting, etc.), and the results were reproduced well in the clinical study.

To further examine the safety aspects of emicizumab prophylaxis and prove its efficacy in lowering ABR in patients with hemophilia A, a series of phase 3 studies, known as HAVEN 1-5 and HOHOEMI, have been planned so far (Table 1). To select the dosing regimens of emicizumab to be tested in these studies, we applied 
a
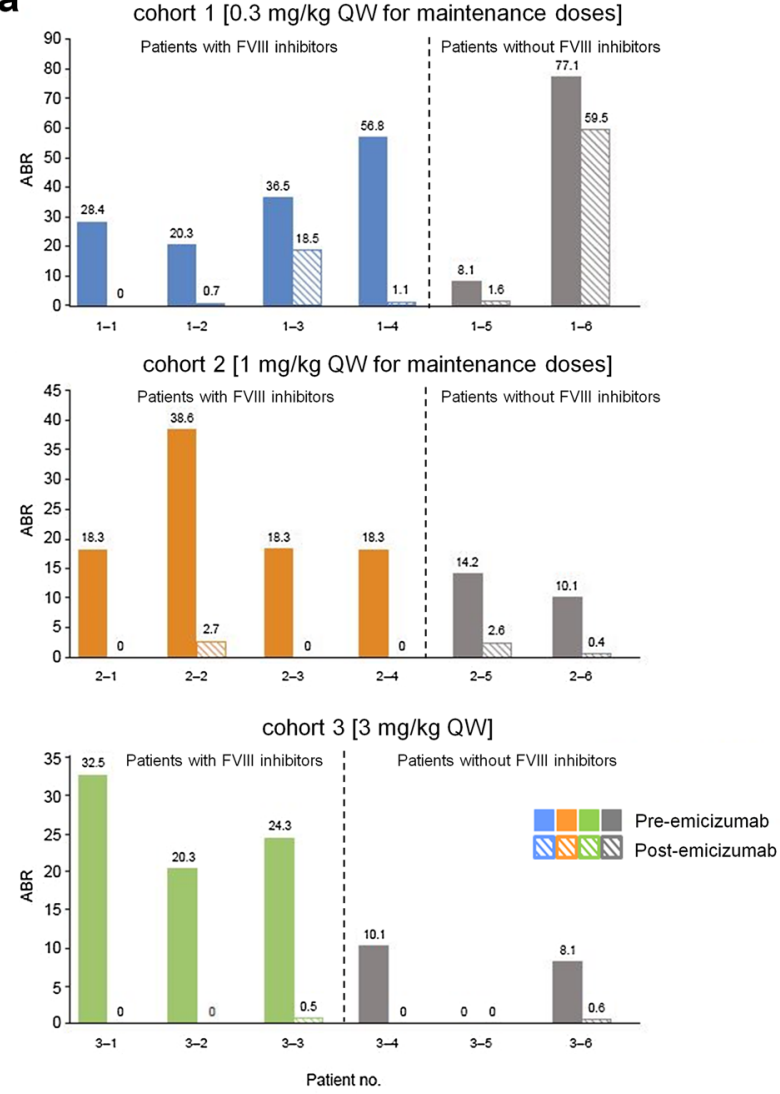

Fig. 4 Efficacy outcomes of the phase 1 and 1/2 studies (combined). a Individual annualized bleeding rates (ABRs) pre- and post-emicizumab for each patient with or without FVIII inhibitors. All data collected following dose up-titrations were excluded; data before dose up-titration were used for $\mathrm{ABR}$ calculation of the combined phase 1

\section{b}

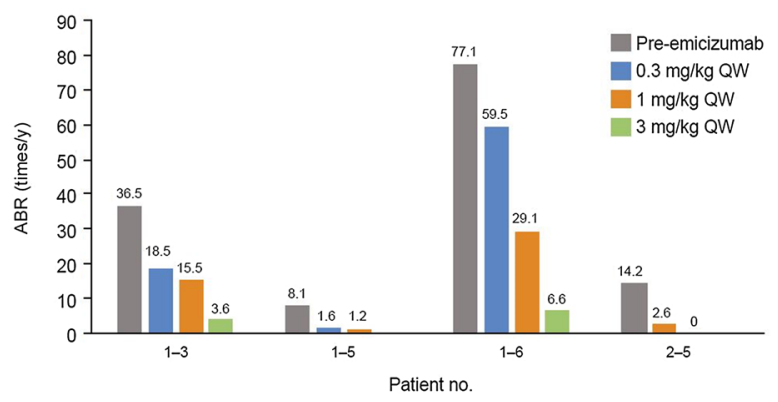

\begin{tabular}{|c|c|c|c|c|}
\hline Dosing & \multicolumn{4}{|c|}{ Weeks to calculate ABR } \\
\hline $0.3 \mathrm{mg} / \mathrm{kg}$ QW & 45 & 96 & 12 & - \\
\hline $1 \mathrm{mg} / \mathrm{kg}$ QW & 27 & 44 & 25 & 81 \\
\hline $3 \mathrm{mg} / \mathrm{kg}$ QW & 72 & - & 72 & 37 \\
\hline
\end{tabular}

(12 weeks) and phase 1/2 extension studies. b Individual ABRs preand post-emicizumab for patients who had dose up-titration. ABRs for each given dose were calculated based on combined data from the phase 1 and $1 / 2$ studies. $Q W$ once weekly. The graphs were quoted and modified from the Supplemental Data of the reference [33]

Table 1 Phase 3 studies of emicizumab

\begin{tabular}{|c|c|c|c|c|c|c|}
\hline \multirow{2}{*}{$\begin{array}{l}\text { Name of Phase } 3 \\
\text { study }\end{array}$} & \multicolumn{3}{|c|}{ Maintenance dosing regimen ${ }^{\mathrm{a}}$} & \multicolumn{3}{|l|}{ Patient population } \\
\hline & Amount & Route & Frequency & Disease & $\begin{array}{l}\text { FVIII } \\
\text { inhibitors }\end{array}$ & Age (body weight) \\
\hline HAVEN 1 & $1.5 \mathrm{mg} / \mathrm{kg}$ & $\mathrm{SC}$ & Every week & Congenital hemophilia A & + & $\geq 12$ years of age $(\geq 40 \mathrm{~kg})$ \\
\hline HAVEN 2 & $\begin{array}{l}1.5 \mathrm{mg} / \mathrm{kg} \\
3 \mathrm{mg} / \mathrm{kg} \\
6 \mathrm{mg} / \mathrm{kg}\end{array}$ & $\begin{array}{l}\mathrm{SC} \\
\mathrm{SC} \\
\mathrm{SC}\end{array}$ & $\begin{array}{l}\text { Every week } \\
\text { Every } 2 \text { weeks } \\
\text { Every } 4 \text { weeks }\end{array}$ & Congenital hemophilia A & + & $\begin{array}{l}<12 \text { years of age, } 12-17 \text { years of age }(<40 \mathrm{~kg}) \\
\geq 2 \text { and }<12 \text { years of age }\end{array}$ \\
\hline HAVEN 3 & $\begin{array}{l}1.5 \mathrm{mg} / \mathrm{kg} \\
3 \mathrm{mg} / \mathrm{kg}\end{array}$ & $\begin{array}{l}\mathrm{SC} \\
\mathrm{SC}\end{array}$ & $\begin{array}{l}\text { Every week } \\
\text { Every } 2 \text { weeks }\end{array}$ & Congenital hemophilia A & - & $\geq 12$ years of age $(\geq 40 \mathrm{~kg})$ \\
\hline HAVEN 4 & $6 \mathrm{mg} / \mathrm{kg}$ & $\mathrm{SC}$ & Every 4 weeks & Congenital hemophilia A & + or - & $\geq 12$ years of age $(\geq 40 \mathrm{~kg})$ \\
\hline HOHOEMI & $\begin{array}{l}3 \mathrm{mg} / \mathrm{kg} \\
6 \mathrm{mg} / \mathrm{kg}\end{array}$ & $\begin{array}{l}\mathrm{SC} \\
\mathrm{SC}\end{array}$ & $\begin{array}{l}\text { Every } 2 \text { weeks } \\
\text { Every } 4 \text { weeks }\end{array}$ & Congenital hemophilia A & - & $<12$ years of age \\
\hline HAVEN 5 & $\begin{array}{l}1.5 \mathrm{mg} / \mathrm{kg} \\
6 \mathrm{mg} / \mathrm{kg}\end{array}$ & $\begin{array}{l}\mathrm{SC} \\
\mathrm{SC}\end{array}$ & $\begin{array}{l}\text { Every week } \\
\text { Every } 4 \text { weeks }\end{array}$ & Congenital hemophilia A & + or - & $\geq 12$ years of age $(\geq 40 \mathrm{~kg})$ \\
\hline
\end{tabular}

$S C$ subcutaneous administration

${ }^{\text {a }}$ The maintenance dosing will start after completing a loading dose of $3 \mathrm{mg} / \mathrm{kg}$ every week for initial 4 weeks 
pharmacometric approaches on the basis of the outcomes of the phase 1 and 1/2 studies, which substituted for a conventional dose-finding study [28, 34].

The results of the HAVEN 1 study were published in 2017 [29]. The study assessed the efficacy, safety, and pharmacokinetics of once-weekly subcutaneous emicizumab prophylaxis in hemophilia A patients with FVIII inhibitors. The primary objective was to compare bleeding rates among participants previously given episodic treatment with bypassing agents who received emicizumab prophylaxis versus no prophylaxis. A total of 109 participants were enrolled. The ABR was 2.9 events (95\% confidence interval [CI] 1.7-5.0) among participants who were randomly assigned to emicizumab prophylaxis ( $n=35$, Group A), versus 23.3 events (95\% CI 12.3-43.9) among those assigned to no prophylaxis $(n=18$, Group B), representing a significant difference of $87 \%$ in favor of emicizumab prophylaxis $(P<0.001)$. A total of 22 participants in Group A $(63 \%)$ had zero bleeding events, as compared with 1 participant $(6 \%)$ in Group B. Among 24 patients in Group $\mathrm{C}$ who had participated in an earlier non-interventional study, emicizumab prophylaxis resulted in ABR that was significantly lower by $79 \%$ than the patient's previous rate of prophylaxis with a bypassing agent $(P<0.001)$. In addition, the emicizumab prophylaxis improved the Physical Health Score of the HaemophiliaSpecific Quality-of-Life (Haem-A-QoL) Questionnaire.

In this HAVEN 1 study, 198 adverse events were reported in 103 participants receiving emicizumab prophylaxis; the most frequent events were injection-site reactions (in 15\% of participants). Five serious thrombotic adverse events were evident, with 3 reports of thrombotic microangiopathy (TMA), 1 skin necrotic superficial thrombophlebitis, and 1 cavernous sinus thrombosis. These complications occurred in patients treated with multiple infusions of aPCC under the emicizumab prophylaxis [29]. The case of one patient who developed TMA was eventually fatal, although the TMA was resolving at the time of death. He received aPCC treatment against rectal hemorrhage for 4 days consecutively.

On the whole, the statistical significance of the outcomes seen in the HAVEN 1 study underwrote the efficacy observed in the phase 1 and 1/2 studies. Emicizumab was approved in US in 2017 and also in EU, Japan, and some other countries in 2018 on the basis of the positive results from this HAVEN 1 study and the interim results from the HAVEN 2 study. In these first approvals, emicizumab was indicated for prophylaxis to prevent or reduce the frequency of bleeding episodes in adult and pediatric patients with hemophilia A (congenital FVIII deficiency) with FVIII inhibitors. Its prescribing information in US includes a boxed warning about TMA and thromboembolism, stating that cases of TMA and thrombotic events were reported when on average a cumulative amount of $>100 \mathrm{U} / \mathrm{kg} / 24 \mathrm{~h}$ of aPCC was administered for $24 \mathrm{~h}$ or more to patients receiving emicizumab prophylaxis.
Most recently, the results of the HAVEN 3 study were published [35]. The study investigated once-weekly or once-biweekly subcutaneous emicizumab prophylaxis in hemophilia A patients without FVIII inhibitors. For the once-weekly maintenance dosing regimen, $1.5 \mathrm{mg} / \mathrm{kg}$ of emicizumab was administered per infusion which was identical to that in the HAVEN 1 and 2 studies. For the once-biweekly maintenance dosing regimen, $3 \mathrm{mg} / \mathrm{kg}$ of emicizumab was administered per infusion. Therefore, despite the difference of the doses per infusion, the two regimens required the identical usage amount of emicizumab on the whole.

A total of 152 participants were enrolled. The ABR was 1.5 events (95\% CI $0.9-2.5$ ) in the once-weekly emicizumab prophylaxis cohort (Group A, $n=36$ ) and 1.3 events (95\% CI 0.8-2.3) in the once-biweekly emicizumab prophylaxis cohort (Group B, $n=35$ ), as compared with 38.2 events (95\% CI 22.9-63.8) in the no prophylaxis cohort (Group C, $n=18$ ); thus, the rate was $96 \%$ lower in Group A and 97\% lower in Group B $(P<0.001$ for both comparisons). In the intra-individual comparison involving 48 patients (Group D) who had participated in an earlier non-interventional study, emicizumab prophylaxis resulted in an ABR that was $68 \%$ lower than the ABR with previous FVIII prophylaxis $(P<0.001)$. The most frequent adverse event was low-grade injection-site reaction. There were no thrombotic or TMA events, or new development of FVIII inhibitors. These results indicated the possibility that the options of emicizumab prophylaxis may be expanded in terms of flexibility in dosing regimens and scope of the patients. (During the proof process of this manuscript, Food and Drug Administration (FDA) of US expanded the regulatory approval of emicizumab: "for routine prophylaxis to prevent or reduce the frequency of bleeding episodes in adults and children with hemophilia A both with and without FVIII inhibitors, administered once weekly, every 2 weeks, or every 4 weeks".)

No neutralizing ADA has been reported in the HAVEN 1 and 3 studies, but a patient in the HAVEN 2 study was recently reported to have developed neutralizing ADAs to emicizumab and his emicizumab prophylaxis was stopped. So far, hundreds of hemophilia A patients have been treated with emicizumab worldwide, but this was the first case of developing problematic neutralizing ADAs during its prophylaxis, whereas inhibitors to FVIII agents (neutralizing ADAs) have developed in as many as $1 / 3$ of the patients with severe hemophilia A [8]. In the case of anti-TNF $\alpha$ biologics, ADAs developed in $12.7 \%$ of the receiving people, and the clinical response was reduced in $67 \%$ of the ADA-positive cases [36]. Thus, emicizumab's record on immunogenicity is considered to have been reasonably favorable so far. 


\section{Biomarkers in the presence of emicizumab}

Finally, in this article, we would like to mention the biomarkers in the patients treated with emicizumab. APTT and APTT-based assays are occasionally used for patients with hemophilia A [2]. However, the action of emicizumab on these assays is intensive in terms of the comparison with that of FVIII. It is because FVIII requires additional time to be activated by thrombin or FXa in these assay systems, whereas emicizumab in nature does not require to be activated by a protease. As a result, emicizumab at the clinical doses interferes with or masks the outcomes of APTT itself and other APTT-based assays including one-stage clotting assays to determine FVIII, FIX, and Protein $\mathrm{C}$ activities and the Bethesda assay to determine FVIII inhibitor titer. We recently advocated a methodology of adding two anti-emicizumab neutralizing antibodies in vitro to eliminate the influence of emicizumab on the plasma-based assays that measure FVIII activity or FVIII inhibitor titer [37]. These two anti-emicizumab neutralizing antibodies were derived from the animals (a mouse and a rat) immunized by ourselves with the respective variable regions of emicizumab. Using this method, FVIII activity and FVIII inhibitor titer can be measured even under emicizumab treatment. Scientifically, chromogenic assays consisting of bovine coagulation factors can also be used for determining FVIII activity and FVIII inhibitor titer under emicizumab treatment, because emicizumab does not exert its cofactor activity on the bovine FIXa-catalyzed bovine FX activation.

The difference in sensitivity to APTT and APTT-based assays between FVIII and emicizumab also raised the issue of how to establish effective laboratory methods to assess hemostatic control in emicizumab-treated patients. In the phase 1 [Part C] and its extension phase $1 / 2$ studies that included 3 emicizumab-dosing groups, a plasma emicizumab concentration-dependent reduction in bleeding frequency was observed (Fig. 4), while there was no clear correlation between bleeding onset and either APTT or the parameters of the thrombin generation assay of a certain condition [28]. Emicizumab concentration in plasma can be an effective option of biomarker to predict hemostatic potential driven by emicizumab. However, it reflects neither the basic condition of a patient nor the effect of other drugs if used in combination. In an attempt to measure the whole coagulation potential of plasma in the presence of emicizumab, we are examining some methodologies, including a modified clot waveform analysis [25].

As above, we have described the limitations of APTT and APTT-based assays in the presence of emicizumab. To avoid doubt, however, APTT is considered to be effective for detecting the possible development of anti-emicizumab neutralizing alloantibodies and for just confirming the presence of emicizumab in the treated patients. Since APTT is widely used, how to use APTT for patients treated with emicizumab should be further explored.

\section{Conclusion}

We reviewed the course of the research and clinical development of emicizumab, which is a humanized anti-FIXa/ FX bispecific IgG antibody with a FVIIIa-cofactor activity, and also described the characteristics of the emicizumab molecule. As described above, emicizumab prophylaxis is expected to reduce the bleeding frequency in patients and the burden on them and their families, as well as improve their quality of life, even when FVIII inhibitors have developed.

The emicizumab molecule is unique and novel. To make the clinical application of emicizumab more valuable, further clinical and non-clinical research is required, including accumulating clinical experience on using emicizumab in combination with other drugs and molecular structural analyses to visualize the mechanisms of action of emicizumab. These attempts will provide good clues to improve both the efficacy and safety of emicizumab prophylaxis.

\section{Compliance with ethical standards}

Conflict of interest MS receives research Grants and/or (consulting) honoraria from Chugai, F. Hoffmann-La Roche, Baxalta, Bayer, BioMarine, Bioverativ, CSL Behring, Novo Nordisk, Pfizer, Sanofi and Sysmex, and is engaged in clinical studies sponsored by Chugai and Roche. TK is an employee of Chugai and possesses Chugai stock. TK and MS are inventors of the patents relating to emicizumab.

\section{References}

1. Geraghty S, Dunkley T, Harrington C, Lindvall K, Maahs J, Sek J. Practice patterns in haemophilia A therapy-global progress towards optimal care. Haemophilia. 2006;12(1):75-81.

2. Srivastava A, Brewer AK, Mauser-Bunschoten EP, Key NS, Kitchen S, Llinas A, et al. Treatment guidelines working group on behalf of the World Federation of Hemophilia. Guidelines for the management of hemophilia. Haemophilia. 2013;19(1):e1-47.

3. Kasper CK, Mannucci PM, Bulyzhenkov V, Brettler DB, Chuansumrit A, Heijnen L, et al. Hemophilia in the 1990s: principles of management and improved access to care. Semin Thromb Hemost. 1992;18(1):1-10.

4. Berntorp E, Dolan G, Hay C, Linari S, Santagostino E, Tosetto $\mathrm{A}$, et al. European retrospective study of real-life haemophilia treatment. Haemophilia. 2017;23(1):105-14.

5. Balkaransingh $P$, Young G. Novel therapies and current clinical progress in hemophilia A. Ther Adv Hematol. 2018;9(2):49-61.

6. Mahlangu J, Cerquiera M, Srivastava A. Emerging therapies for haemophilia-global perspective. Haemophilia. 2018;24(Suppl 6):15-21.

7. Shi Q, Kuether EL, Schroeder JA, Fahs SA, Montgomery RR. Intravascular recovery of VWF and FVIII following 
intraperitoneal injection and differences from intravenous and subcutaneous injection in mice. Haemophilia. 2012;18(4):639-46.

8. Peyvandi F, Mannucci PM, Garagiola I, El-Beshlawy A, ElaIfy M, Ramanan V, et al. A randomized trial of factor VIII and neutralizing antibodies in hemophilia A. N Engl J Med. 2016;374(21):2054-64.

9. Hay CR, DiMichele DM. The principal results of the International Immune Tolerance Study: a randomized dose comparison. Blood. 2012;119(6):1335-44

10. Kitazawa T, Igawa T, Sampei Z, Muto A, Kojima T, Soeda T, et al. A bispecific antibody to factors IXa and $\mathrm{X}$ restores factor VIII hemostatic activity in a hemophilia A model. Nat Med. 2012;18(10):1570-4.

11. George LA. Hemophilia gene therapy comes of age. Blood Adv. 2017;1(26):2591-9.

12. Sampei Z, Igawa T, Soeda T, Okuyama-Nishida Y, Moriyama $\mathrm{C}$, Wakabayashi $\mathrm{T}$, et al. Identification and multidimensional optimization of an asymmetric bispecific IgG antibody mimicking the function of factor VIII cofactor activity. PLoS One. 2013;8(2):e57479.

13. Lenting PJ, Donath MJ, van Mourik JA, Mertens K. Identification of a binding site for blood coagulation factor IXa on the light chain of human factor VIII. J Biol Chem. 1994;269(10):7150-5.

14. Fay PJ, Koshibu K. The A2 subunit of factor VIIIa modulates the active site of factor IXa. J Biol Chem. 1998;273(30):19049-54.

15. Lapan KA, Fay PJ. Localization of a factor $X$ interactive site in the A1 subunit of factor VIIIa. J Biol Chem. 1997;272(4):2082-8.

16. Fay PJ. Factor VIII structure and function. Int $\mathbf{J}$ Hematol. 2006;83(2):103-8.

17. Yegneswaran S, Wood GM, Esmon CT, Johnson AE. Protein S alters the active site location of activated protein $\mathrm{C}$ above the membrane surface. A fluorescence resonance energy transfer study of topography. J Biol Chem. 1997;272(40):25013-21.

18. Soeda T, Nogami K, Nishiya K, Takeyama M, Ogiwara K, Sakata Y, et al. The factor VIIIa C2 domain (residues 2228-2240) interacts with the factor IXa Gla domain in the factor Xase complex. J Biol Chem. 2009;284(6):3379-88.

19. Takeyama M, Wakabayashi H, Fay PJ. Factor VIII light chain contains a binding site for factor $\mathrm{X}$ that contributes to the catalytic efficiency of factor Xase. Biochemistry. 2012;51(3):820-8.

20. Saphire EO, Stanfield RL, Crispin MD, Parren PW, Rudd PM, Dwek RA, et al. Contrasting IgG structures reveal extreme asymmetry and flexibility. J Mol Biol. 2002;319(1):9-18.

21. Stoilova-McPhie S, Villoutreix BO, Mertens K, Kemball-Cook G, Holzenburg A. 3-Dimensional structure of membrane-bound coagulation factor VIII: modeling of the factor VIII heterodimer within a 3-dimensional density map derived by electron crystallography. Blood. 2002;99(4):1215-23.

22. Merchant AM, Zhu Z, Yuan JQ, Goddard A, Adams CW, Presta $\mathrm{LG}$, et al. An efficient route to human bispecific IgG. Nat Biotechnol. 1998;16(7):677-81.

23. Kitazawa T, Esaki K, Tachibana T, Ishii S, Soeda T, Muto A, et al. Factor VIIIa-mimetic cofactor activity of a bispecific antibody to factors IX/IXa and X/Xa, emicizumab, depends on its ability to bridge the antigens. Thromb Haemost. 2017;117(7):1348-57.

24. Muto A, Yoshihashi K, Takeda M, Kitazawa T, Soeda T, Igawa T, et al. Anti-factor IXa/X bispecific antibody (ACE910): hemostatic potency against ongoing bleeds in a hemophilia A model and the possibility of routine supplementation. J Thromb Haemost. 2014;12(2):206-13.

25. Nogami K, Matsumoto T, Tabuchi Y, Soeda T, Arai N, Kitazawa $\mathrm{T}$, et al. Modified clot waveform analysis to measure plasma coagulation potential in the presence of the anti-factor IXa/ factor X bispecific antibody emicizumab. J Thromb Haemost. 2018;16(6):1078-88.

26. Muto A, Yoshihashi K, Takeda M, Kitazawa T, Soeda T, Igawa $\mathrm{T}$, et al. Anti-factor IXa/X bispecific antibody ACE910 prevents joint bleeds in a long-term primate model of acquired hemophilia A. Blood. 2014;124(20):3165-71.

27. Shima M, Hanabusa H, Taki M, Matsushita T, Sato T, Fukutake $\mathrm{K}$, et al. Factor VIII-mimetic function of humanized bispecific antibody in hemophilia A. N Engl J Med. 2016;374(21):2044-53.

28. Yoneyama K, Schmitt C, Kotani N, Levy GG, Kasai R, Iida S, et al. A pharmacometric approach to substitute for a conventional dose-finding study in rare diseases: example of phase III dose selection for emicizumab in hemophilia A. Clin Pharmacokinet. 2017;57(9):1123-34.

29. Oldenburg J, Mahlangu JN, Kim B, Schmitt C, Callaghan MU, Young G, et al. Emicizumab prophylaxis in hemophilia A with inhibitors. N Engl J Med. 2017;377(9):809-18.

30. Noguchi-Sasaki M, Soeda T, Ueyama A, Muto A, Hirata M, Kitamura $\mathrm{H}$, et al. Emicizumab, a bispecific antibody to factors IX/IXa and $\mathrm{X} / \mathrm{Xa}$, does not interfere with antithrombin or TFPI activity in vitro. TH Open. 2018;2(1):e96-103.

31. Yada K, Nogami K, Shinozawa K, Kitazawa T, Hattori K, Amano K, Fukutake K, Shima M. Emicizumab-mediated hemostatic function in patients with hemophilia $\mathrm{A}$ is down regulated by activated protein $\mathrm{C}$ through inactivation of activated factor V. Br J Hematol. 2018. https://doi.org/10.1111/bjh.15525. (Epub ahead of print).

32. Uchida N, Sambe T, Yoneyama K, Fukazawa N, Kawanishi T, Kobayashi S, et al. A first-in-human phase 1 study of ACE910, a novel factor VIII-mimetic bispecific antibody, in healthy subjects. Blood. 2016;127(13):1633-41.

33. Shima M, Hanabusa H, Taki M, Matsushita T, Sato T, Fukutake $\mathrm{K}$, et al. Long-term safety and efficacy of emicizumab in a phase $1 / 2$ study in patients with hemophilia A with or without inhibitors. Blood Adv. 2017;1(22):1891-9.

34. Yoneyama K, Schmitt C, Chang T, Levy GG. Model-informed dose selection for pediatric study of emicizumab in hemophilia A. Clin Pharmacol Ther. 2018;103(Suppl 1):94.

35. Mahlangu J, Oldenburg J, Paz-Priel I, Negrier C, Niggli M, Mancuso ME, et al. Emicizumab prophylaxis in patients who have hemophilia A without inhibitors. N Engl J Med. 2018;379(9):811-22.

36. Thomas SS, Borazan N, Barroso N, Duan L, Taroumian S, Kretzmann B, et al. Comparative immunogenicity of TNF inhibitors: impact on clinical efficacy and tolerability in the management of autoimmune diseases. A systematic review and meta-analysis. BioDrugs. 2015;29(4):241-58.

37. Nogami K, Soeda T, Matsumoto T, Kawabe Y, Kitazawa T, Shima M. Routine measurements of factor VIII activity and inhibitor titer in the presence of emicizumab utilizing anti-idiotype monoclonal antibodies. J Thromb Haemost. 2018;16(7):1383-90. 